\title{
Hybrid Geo-Location Routing Protocol for Indoor and Outdoor Positioning Applications
}

\author{
Sania Mushtaq ${ }^{1}$, Dr. Gasim Alandjani ${ }^{2}$, Saba Farooq Abbasi ${ }^{3}$, Dr. Nasser Abosaq ${ }^{4}$ \\ Dr. Adeel Akram ${ }^{5}$, Dr. Shahbaz Pervez ${ }^{6}$ \\ Dept. of Telecom. Engineering, University of Engg. and Technology, Taxila, Pakistan ${ }^{1,3,5}$ \\ CSE Department, Yanbu University College, Kingdom of Saudi Arabia ${ }^{2,4}$ \\ Abacus Institute of Studies, Christchurch, New Zealand ${ }^{6}$
}

\begin{abstract}
Internet of Things (IoT) essentially demands smart connectivity and contextual awareness of current networks with low power and cost effective wireless solutions. Routing is the backbone of the system controlling the flow of transmission. This work demonstrates a collation between performance investigations of a location-based routing protocol Geocast Adaptive Mesh Environment for Routing with contextual information collected from Global Positioning System (GPS) and Framework for Internal Navigation and Discovery (FIND) respectively. The systems are evaluated based on various metrics i.e. Accuracy, Packet Delivery Ratio and Packet Overhead by means of Network Simulator (NS-2). FIND shows enhanced performance in most cases as compared to GPS for indoor and outdoor environments. The results of this research can be deployed in different areas such as in-building navigation, hospital patient tracking, Smart City context aware service provisioning and Industry 4.0 deployments.
\end{abstract}

Keywords-Internet of Things (IoT); Network Simulator (NS2); Routing; Geocast Adaptive Mesh Environment for Routing (GAMER); Mobility; Global Positioning System (GPS); Framework for Internal Navigation and Discovery (FIND)

\section{INTRODUCTION}

Historically, the wireless networks started during the 1970s and their significance has been growing subsequently since. An Ad hoc Network is a temporary network without any wires, specific infrastructure and administrative intervention. A MANET consist of wireless mobile nodes that formulates a system short of communication frame or standard support services. MANETs are considered as the next generation of networks. Two hosts in a MANET may route their traffic using hops through other hosts in the network [1]. Unpredictable topology variation can be caused due to host mobility. Therefore, discovering and sustaining routes in a MANET is substantial [2].

IoT is briskly accelerating into the technological world by linking the everyday objects to Internet. The significance of an emerging area like IoT in technical, economic and social fields is exceptional. This concept is still considered largely theoretical on one hand but also a network that is used every single day on the other. The effect of IoT in our everyday life primarily incorporates transportation, health and exercise, home (smart home), business, pollution and waste management, etc. [3]. Intelligent and self-configuring nodes (things) interconnected in an influential and global network framework defines the IoT prototype. It serves as one of the most disruptive technologies, presenting practical scenarios for ubiquitous and pervasive computing. Real world and small things with substantial concerns with respect to performance, privacy, security and reliability along with limited processing and storage capacity generally defines IoT [4]. This revolutionary idea gives indication to the possibility of smart cities, campuses, health care systems etc.

An Ad-Hoc wireless network comprises of a group of dynamically and randomly dispersed mobile nodes that are independent of permanent structure as opposed to IoT. Nevertheless, excessive similarities among node movements have been observed between IoT and Ad-Hoc networks [5]. For effective transmission between nodes, a range of existing Ad-Hoc routing protocols have been researched and subjugated.

Geocast Routing takes advantage of the contextual information for routing. Routing of information (from source to destination) is the significant part of any IoT and wireless sensor network (WSN) solutions. Multi-hop transmissions are the basis of node topology and has been utilized in healthcare, environment monitoring and many other smart systems [6]. By utilizing location information for mobile hosts, overhead of route discovery can be minimized. This location data is typically assimilated through GPS. However, the cost, size and power requirement of the sensor devices surges due to the addition of GPS devices. GPS is not suitable for indoor applications and results in additional power intake [7].

For acquiring efficient routing numerous routing protocols have been envisioned. When hosts move, these protocols vary in the technique adopted for finding a new route and/or modifying an existing one. However, one of the grass root problems in mobile computing is localization of a device [8]. The design and implementation of location aware systems became conceivable due to the importance and need of location information. Failure of Global Positioning System (GPS) in indoor and urban environments played a fundamental role in the development of indoor location dependent systems.

Geocoding is the process of converting human-readable addresses into machine-process able geographic co-ordinates (i.e. longitude and latitude). When working with data usually there are human-readable location names (e.g. London) but for a computer to process the location geographical coordinates (e.g. longitude $=51.745$, latitude $=-0.81$ ) are needed provided 
by geocoding process. Geocoding needs a database containing listed names of places and their corresponding geographical coordinates. Various open source databases such as geo-names and Open Street Map are available on the internet.

FIND is a simplified way to achieve indoor positioning. It lets us gather indoor location information by our computer (Wi-Fi enabled) or smartphones. Location can easily be discovered inside the homes (bedroom, kitchen, living room etc.) or offices [9]. It was designed with an aspiration to replace motion sensors. Therefore, because of its high resolution, it can replace any motion sensors deployed. It can supply explicit location and user-particular information. FIND can be used as an information source for operations performed through the data collected by motion sensors and GPS. However, apart from this, it provides indoor positioning making the overall system more accurate than the systems mentioned. The geolocation accuracy of FIND is below $10 \mathrm{sq}$. ft., which puts it way ahead of GPS. FIND backs information reception from any source and has integral passive scanning operation [10]. The geocast data acquired through FIND may be used from minimal lifestyle tracking and household automation to extensive commercial applications.

The remaining paper is formulated as below. Section 2 clarifies Geocast Protocols and their categories. Section 3 enlightens the Geocoding and its available sources. Section 3 also administers an outline of routing protocol used in the study. Section 4 and 5 describe the simulation environment and performance parameters while Section 6 contains the results obtained from simulation trials. Finally, conclusion of the paper is enclosed in Section 7.

\section{GeOcAst PRotocols}

Geocast Routing makes use of the contextual information i.e. Location of sensor nodes. For nodes in close proximity, location information is dispatched through signal strength. While for distant nodes, location information can be addressed through information exchanged between neighboring nodes. One of the leading limitations for efficient data transmission is power consumption. Sleep mode of nodes tends to decrease the power consumption [11]. Contrary to other protocols (where the data packet is transmitted to a target node within a network), the destination will be a geographic region provided by geocast group.

These protocols include Flooding [12], Voronoi (Voronoi diagram based geo-casting protocol) [12], LBM (LocationBased Multicast) [13], GAMER (Geocast Adaptive Mesh Environment for Routing) [14]. Geo-GRID [15], URAD (Unicast Routing with Area Delivery) [16] and TORA (Temporally Ordered Routing Algorithm) [17]. The main comparative characteristics between geocast protocols are enlisted in Table I.

A flooding-based LBM, routing based GAMER and cluster based Geo-GRID protocols have been studied and compared in [18] based on node mobility, node speed and node density. Although Geo-GRID has a substantial performance in compact environment as compared to GAMER and LBM, GAMER triumphs in packet overhead with the lowest value.
TABLE. I. COMPARISON OF GEOCAST ROUTING PROTOCOLS

\begin{tabular}{|l|l|l|l|}
\hline Protocol & $\begin{array}{l}\text { Type of } \\
\text { Routing }\end{array}$ & Scalability & $\begin{array}{l}\text { Memory } \\
\text { Requirement }\end{array}$ \\
\hline Flooding [12] & Flooding & Low & No \\
\hline Voronoi [12] & Flooding & Medium & Low \\
\hline LBM [13] & Flooding & Medium & Low \\
\hline GAMER [14] & Routing & $\begin{array}{l}\text { Medium } \\
\text { High }\end{array}$ & Medium \\
\hline Geo-GRID [15] & Flooding & Low High & Low \\
\hline URAD [16] & Routing & $\begin{array}{l}\text { Medium } \\
\text { High }\end{array}$ & Low \\
\hline TORA [17] & Routing & High & Medium \\
\hline
\end{tabular}

Reference [19] discusses the performance of GAMER, Geo-GRID and Geo-TORA. The study is based on the selection of geocast area and efficient routing of these protocols. GAMER and Geo-GRID follow a mesh of routes between source and destination in a geocast area. However, Geo-TORA follows unicasting followed by flooding in the geocast region for routing.

\section{GAMER OVERVIEW}

A guide-based protocol GAMER is selected for the comparative study and was developed by the researchers as mentioned in [20]. This is a position centered routing protocol for ad hoc networks. A fusion of flooding and greedy protocols makes up this approach. A web of routes is sustained between the transmitting node and destination region in a highly scalable routing protocol. The excessive paths present in the mesh are available in case of link failure occurring due to dynamic topology in ad hoc networks. The suitable path for packet forwarding is determined via link interval of the feedback at each node. This ensures the delivery of data packets in this approach. Conversely, an idleness of relay nodes in the forwarding area and an extensive endwise delay is fashioned. Furthermore, if a forwarding node does not have adjacent nodes in the route of targeted node the dispatch of data packets in that way remains incomplete [21].

Inside a forwarding zone, JOIN-DEMAND (JD) packets are flooded to establish the mesh. A JT packet is produced and unicasted back to the transmitting node by receiving node inside geocast region after the reception of a non-replicate JD packet. The path taken by JT packet is the same followed by JD packet previously. The mesh makes each node used for reverse routing its member. The mesh members circulate the geocast packets produced by source node within mesh and flood them inside the target area.

GAMER prefers FA (Forwarding Approach) to minimize the load as much as it can and changes the complexity of the mesh effectively. GAMER operates on adaptation and changes it forwarding area to a smaller size if the existing one thrives and to a larger size if it declines. There are three types of FAs a node in GAMER can pick from i.e. CONE CORRIDOR and FLOOD. First type of FA is called FLOOD, which floods the JD packets all through the network (ad hoc). A forwarding area is established in order to minimize the flooding area for Join Demand packets in the remaining two types of FA. Mobile Networks (MN) inside the forwarding zone are capable of 
flooding the JD packets exclusively. In CORRIDOR approach, a rectangle shaped forwarding zone is produced by considering the region between two parallel lines curved around the geocast region. In CONE FA, forwarding zone in a mesh is the area confined by an angle. Source node is at the vertex of this angle while geocast region is the area present between its sides. In comparison, the forwarding zone in FLOOD forwarding area is much bigger than forwarding area in CORRIDOR FA [14]. The mesh created by CONE FA has even more confined forwarding zone relative to mesh created by CORRIDOR FA.

The transitional nodes do not need to be aware of or forward the routing state of other neighboring nodes in GAMER, which is considered as its main interest. On the other hand, importing the complete route from source to the target by data packets results in increased overhead, adding to the drawbacks.

\section{Simulation EnVIRONMENT}

The network simulator NS-2 [22] is used to implement geocast protocol, distinctly prominent in ad hoc network community. The simulation region is rectangle shaped with $300 \times 600 \mathrm{~m}$ dimensions. The Geocast region is in the form of $150 \times 150 \mathrm{~m}$ square positioned at the upper right corner. A stationary node is placed at the center of Geocast region to guarantee the presence of one node for reception of transmitted geocast packets. Transmission Range for each Mobile Node $(\mathrm{MN})$ is uniform i.e. 100 meters each mobile node has the link bandwidth of 2 Mbps. A 64-byte geocast data packet is generated by a single CBR (Constant Bit Rate) source for every 1000-second simulation period. To bypass unneeded collisions, a uniform random jitter is introduced in packet scheduling.

Random waypoint mobility model is used for movement of nodes [23]. In this model, each node grows from a random starting point to a randomly picked target. The node takes a rest period at the target and picks another random destination after the pause time. This cycle is replicated throughout the simulation period resulting in regular topology changes of the network. [24] Suggests that for a random waypoint model, location initialization and Mobile Node pause time is established with the steady state distribution to escape the initialization difficulty of the model.

TABLE. II. SIMULATION PARAMETERS [25]

\begin{tabular}{|l|l|}
\hline Parameter & Value \\
\hline Simulator & NS-2 \\
\hline Protocol & GAMER \\
\hline Simulation Area & $300 \times 600 \mathrm{~m}^{2}$ \\
\hline Simulation Time & $200 \mathrm{sec}$ \\
\hline Geocast Region & $150 \times 150 \mathrm{~m}^{2}$ \\
\hline Transmission Range & $100 \mathrm{~m}$ \\
\hline Data Payload & Bytes/packet \\
\hline Link Capacity & 2 Mbps \\
\hline Traffic & Constant Bit Rate \\
\hline Node Movement Model & Random waypoint \\
\hline
\end{tabular}

The summary of model parameters adopted in the simulation trials are specified in Table II.

It is necessary for the data packets to either find a geocast region or expire during the trials. Partition less initialization and timely execution of simulation trials is achieved for this purpose. During simulation trials, data packets are sent with 1 second delay to allow time for formation of mesh and grid. Several simulation trials are performed to present average results.

\section{EVALUATION PARAMETERS}

\section{A. Accuracy}

Accuracy of a system is the indication of proximity of a calculated quantity to a standard quantity. An error ratio between the measured values to the possible range of values is also accounted as accuracy. For location aware systems, the accuracy is determined in terms of distance. It is the difference between the position found by the system and the actual position.

\section{B. Packet Overhead}

Packet Overhead is the amount of time taken for a transmission over a packet - switched network. It is a type of information added to packet header to assure packet delivery to the destination. At the time of network simulation, the overhead is essentially the additional bytes transmitted.

During entire simulation interval i.e. transmission from source to destination nodes, all the packets transmitted are numbered. These labels are usually known as packet overhead. These include any supplementary transmissions taking place for routing data packets i.e. acknowledgements, hello message, control messages etc.

It is considered as the wasted bandwidth as it decreases the comprehensive transmission speed of a data packet.

\section{Packet Delivery Ratio}

PDR is a ratio among the amount of message packets directed by the source and the amount of message packets acknowledged by the target.

The efficiency of the system is directly proportional to the PDR value of that system. The higher the PDR value, the higher the efficiency of the system. PDR of a system can be calculated by a simple formula as below:

\section{$\mathrm{PDR}=\mathrm{Pr} / \mathrm{Pt}$}

In above equation Pr represents the data packets received by the target node while Pt shows the data packets sent by the source node.

\section{RESUlTS AND Discussions}

For this simulation, mesh created through CONE has been chosen. The mesh created by CONE FA has a narrow forwarding zone relative to mesh created by other techniques. Results for GPS based system have been obtained from [20] and are then compared to the results for FIND based system. The original node positions, their accuracy and forwarding area is presented in Fig. 1. 


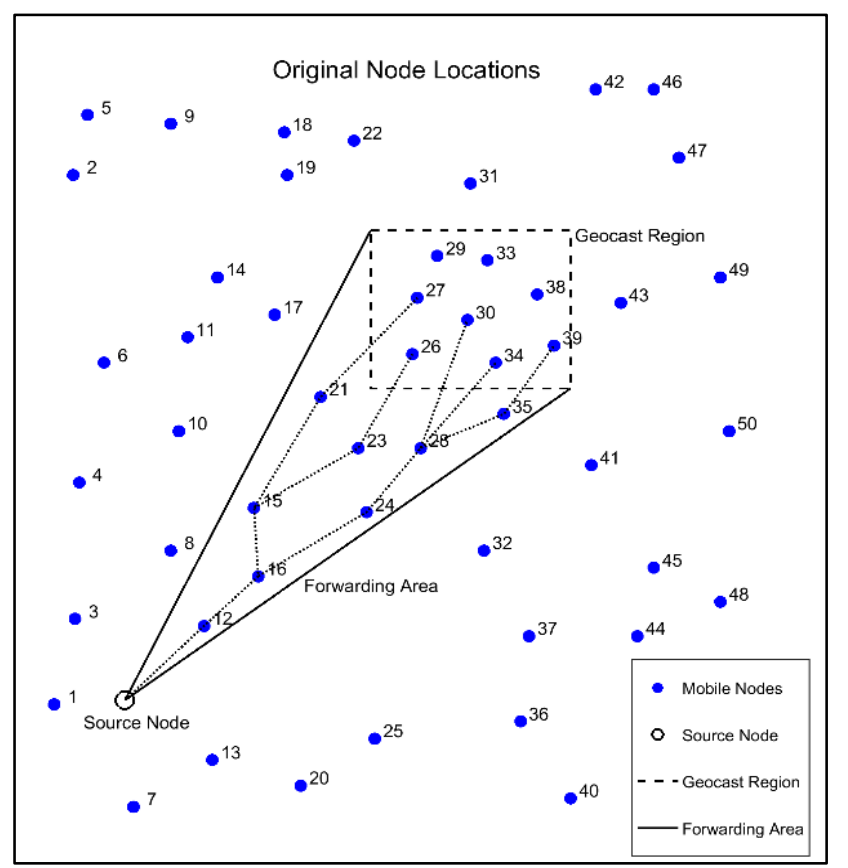

Fig. 1. Forwarding Area for Original Node Locations

\section{A. Accuracy}

The accuracy is one of the most important parameters for a location-based system. It is clearly seen from Fig. 2 the node positions provided by two different information systems i.e. FIND and GPS are different. Fig. 2 displays most of the node positions accurately found through FIND. This is due to the high geolocation accuracy of FIND i.e. 10 sq. feet. The forwarding area selected by using FIND contains accurate node positions, which makes the transmission and delivery of a data packet straightforward.

Fig. 2 also presents the node locations provided through GPS. It is clear that not all the node positions are accurate. This is the result of low GPS accuracy i.e. $3-5 \mathrm{~m}$. The forwarding area selected by using GPS contains some of the inaccurate nodes. This results in low and complicated packet transmission and delivery.

Fig. 3 and 4 show the variation of node locations reported by FIND and GPS systems in comparison to original node locations for indoor and outdoor environments respectively. The $\mathrm{x}$-axis shows each node numbers while $\mathrm{y}$-axis shows the distance error from original positions. The original nodes in blue are taken as a reference and considered at origin i.e. zero axis position. The dashed lines show the error margin of these nodes. The nodes in green are FIND based nodes while nodes in red are GPS based nodes.

It is quite clear from the graphs that GPS operates better in outdoor environments providing greater precision due to the larger distance between nodes. As for indoor environments, GPS shows higher inaccuracy due to the smaller distance between nodes. This behavior results in an inefficient system.

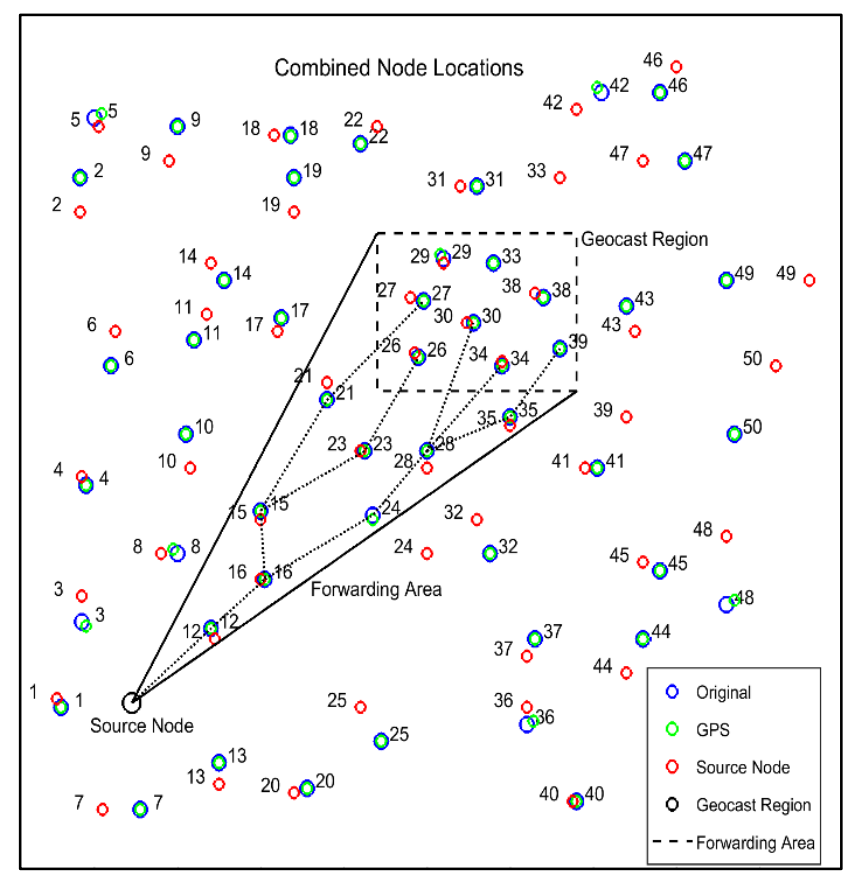

Fig. 2. Combined Node Locations (FIND and GPS).

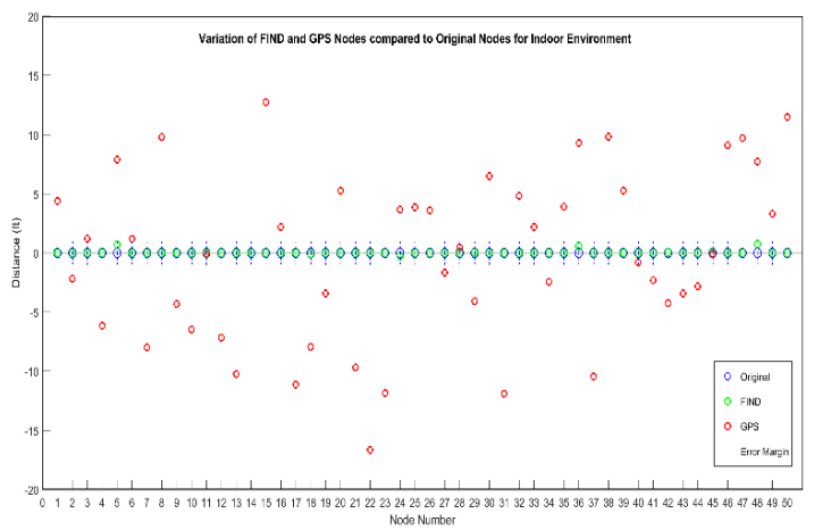

Fig. 3. Variation of FIND and GPS Nodes Compared to Original Nodes for Indoor Environment.

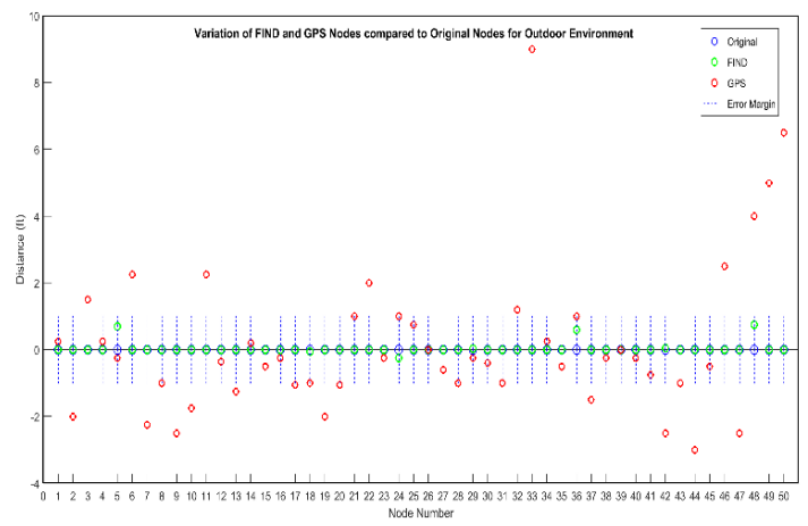

Fig. 4. Variation of FIND and GPS Nodes Compared to Original Nodes for Outdoor Environment. 


\section{B. Packet Overhead Vs Number of Nodes}

Fig. 5 demonstrates Packet Overhead per one success with respect to Number of Nodes for both systems. For simplicity the first analysis is accomplished with static nodes and Null MAC. The number of nodes are increased step by step from 30 to 120 . The source packet rate is kept constant at 40 packets per sec. But due to Null MAC the packet source rate has no effect on the Packet Overhead of the system. The delivery of packets in this case is almost 100 percent.

The result shows that GAMER is very economical in case of Packet Overhead. With the increase in number of nodes Packet Overhead for both cases increases. However, the Packet Overhead per One Success of GAMER is much lower with contextual information obtained through FIND in comparison to information acquired through GPS. This is due to the fact that location information delivered by GPS is in the form of latitude and longitude. The data packet is at least 24 bytes long which results in enlarged Packet Overhead for the system operating on GPS. However, location information delivered by FIND is geocoded and can easily be sent in maximum 8 bytes decreasing the Packet Overhead of the system. Therefore, the system based on FIND shows better performance and is less prone to error than GPS based system.

A clear increase in Packet Overhead for both systems is observed when then number of nodes crosses 80 . The reason for this increase is the increase in grid density i.e. Grids have more than one nodes when the number of nodes exceeds 80 .

\section{Packet Delivery Ratio Vs Source Packet Rate}

Fig. 6 shows the graph between Packet Delivery Ratio and Source Packet Rate. In this study the PDR is calculated while varying Source Packet Rate from 1-80 packets/sec. The number of nodes is set at 80 . The network is kept static with 802.11 MAC. One parameter is varied to efficiently observe the behavior of the system. The system is kept static to exclude the effect of mobility. It is carried out to observe the effect of congestion over a network.

PDR has an inverse relationship with source packet rate. The PDR is almost $100 \%$ for source packet rate up to 30 pkts/sec. Nevertheless, both the systems start showing a decrease in the PDR with further increase in source packet rate. However, the behavior of the FIND based system is much better than the GPS based system according to Fig. 6 .

\section{Packet Overhead Vs Source Packet Rate}

Packet Overhead for a static network with 802.11 MAC is shown in Fig. 7. The system parameters are kept the same as in previous analysis. GAMER has a periodic control overhead which results in no significant change in Packet Overhead with increasing Source Packet Rate. However, it does have a downside i.e. really high packet overhead for low packet rates. For both FIND and GPS based systems GAMER follows the same behavior i.e. Packet Overhead is extremely high for low packet rates, stays the same for medium rates and increases when the network becomes crowded. However, considering the larger size of a GPS Packet and performance of both the systems FIND has a superior performance than a GPS based system.

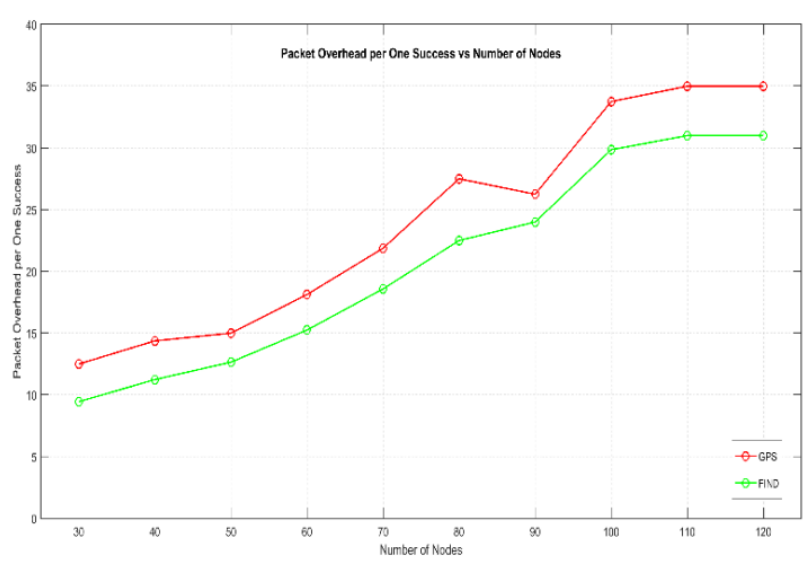

Fig. 5. Packet Overhead vs Number of Node.

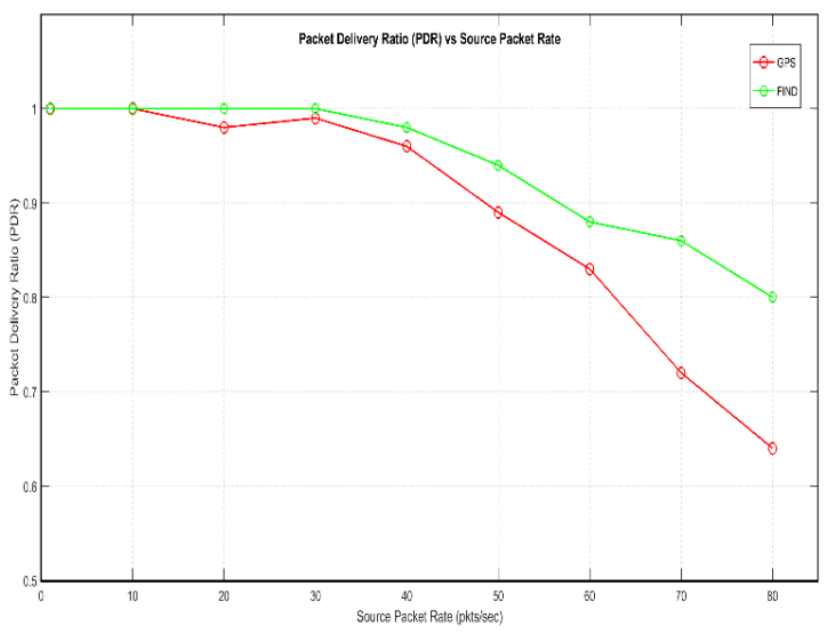

Fig. 6. PDR vs Source Packet Rate.

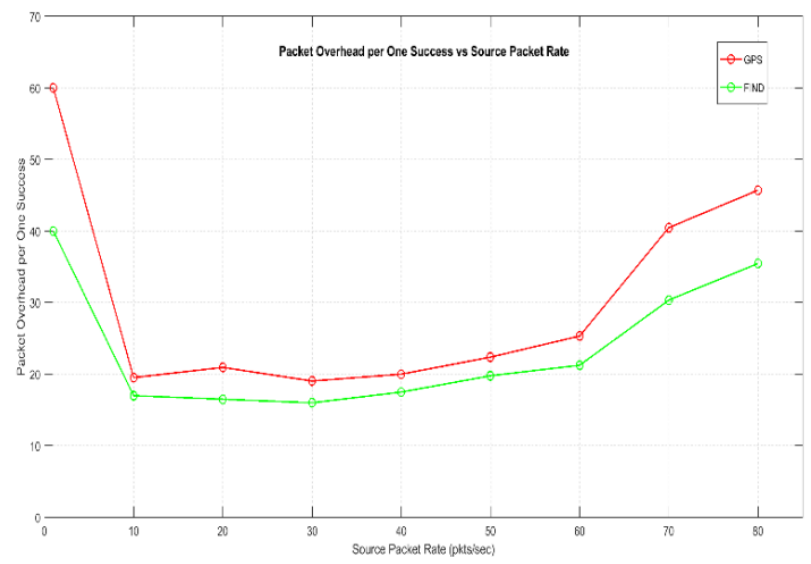

Fig. 7. Packet Overhead vs Source Packet Rate

\section{E. Packet Delivery Ratio Vs Average Node Speed}

The next result is based on mobile nodes to observe the effect of mobility on the systems. The system parameters such as source packet rate and number of nodes are set at 40pkts/sec and 80, respectively. To avoid the effects of traffic Null MAC is used same as first case. PDR is noticed with differing average node speed from $1-20 \mathrm{~m} / \mathrm{s}$. 
The performance of both the systems remains well i.e. greater than $97 \%$ in most cases. GAMER has the tendency to drop the performance with increasing speed. This fact is seen prominently in Fig. 8. However, the performance decline in FIND based system is far less than GPS based system. The delivery ratio in FIND based system is higher due to better accuracy of the nodes contained in the forwarding area.

\section{F. Packet Overhead vs Average Node Speed}

Packet Overhead vs Average Node Speed is presented in Fig. 9. The system parameters are the same as the previous section. It was already discussed in section $B$ that GAMER has a low packet overhead for higher number of nodes. Therefore, even for mobile networks the packet overhead for single success is low as well.

The need to maintain a mesh for transmission is often the reason for increase in packet overhead. The movement of mobile nodes makes it difficult to maintain a mesh throughout the transmission resulting in increased overhead. Although due to inaccuracy of GPS, the incorrect node positions puts the packet overhead level even higher for GPS based GAMER in comparison to FIND based GAMER system.

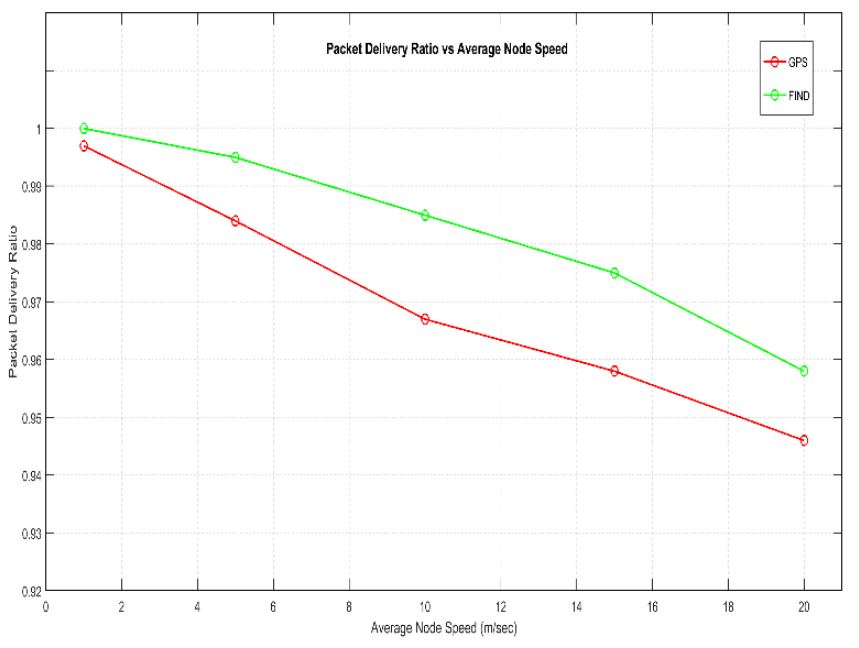

Fig. 8. PDR vs Node Speed.

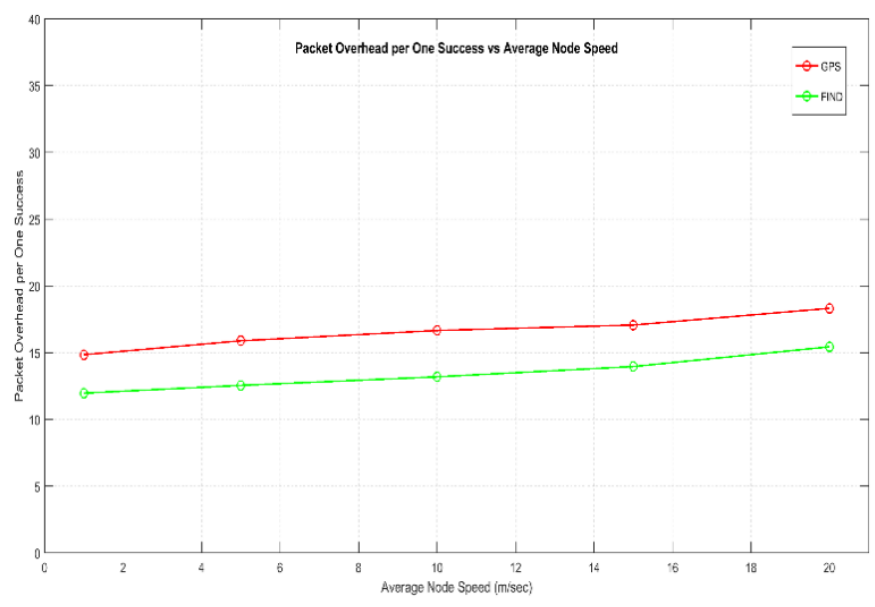

Fig. 9. Packet Overhead vs Node Speed.

\section{CONCLUSION AND FUTURE WORK}

The stated research analyzed a location-based routing protocol GAMER for ad hoc networks based on the two different geolocation systems i.e. GPS and FIND. The performance analysis was centered on Accuracy, Packet Delivery Ratio (PDR) and Packet Overhead of the system. The evaluation discloses that FIND based GAMER performs better in most cases. Results obtained are better than GPS in both indoor and outdoor environment (with mobility). Accuracy of FIND is below 10 sq. ft. as compared to GPS which has an accuracy up to a few meters. To study effects of congestion static nodes are considered. PDR and Packet Overhead of FIND based GAMER shows remarkable results against source packet rate (pkts/sec). Another study carried out considers mobility in a network. The performance of both systems declines as compared to static node systems. PDR of FIND based GAMER remains above $90 \%$ for most cases. However, GPS based GAMER loses the performance when the node speed surpasses $10 \mathrm{~m} / \mathrm{sec}$. A third analysis was conducted by introducing congestion as well as mobility into the network. The performance of FIND system remains superior to GPS systems in all aspects.

In case of FIND system, signals from three or more sources are required for accurate results by FIND fingerprinting. For this purpose, the concept of BYOD (bring your own device) may be used. The information provided by the personal devices can be used rather than carrying around an extra beacon. The battery consumption is almost none due to the use of predefined operations. Additionally, easy installation and configuration of FIND compared to other platforms makes it an ideal replacement. Taking this analysis into account, FIND based GAMER can easily replace any indoor positioning system in use.

\section{REFERENCES}

[1] Rath, Mamata, B. Pattanayak, and Bibudhendu Pati. "A contemporary survey and analysis of delay and power based routing protocols in MANET." ARPN Journal of Engineering and Applied Sciences 11, no. 1 (2016): 536-540.

[2] Singal, Gaurav, Vijay Laxmi, Manoj Singh Gaur, and Vijay Rao. "Moralism: mobility prediction with link stability based multicast routing protocol in MANETs." Wireless Networks 23, no. 3 (2017): 663-679.

[3] Al-Fuqaha, Ala, Mohsen Guizani, Mehdi Mohammadi, Mohammed Aledhari, and Moussa Ayyash. "Internet of things: A survey on enabling technologies, protocols, and applications." IEEE communications surveys \& tutorials 17, no. 4 (2015): 2347-2376.

[4] Botta, Alessio, Walter De Donato, Valerio Persico, and Antonio Pescapé. "On the integration of cloud computing and internet of things." In Future internet of things and cloud (FiCloud), 2014 international conference on, pp. 23-30. IEEE, 2014.

[5] Xin, Hua-Mei, and Kun Yang. "Routing protocols analysis for Internet of Things." In 2015 2nd International Conference on Information Science and Control Engineering, pp. 447-450. IEEE, 2015.

[6] Lee, Tsung-Han, Xiang-Shen Xie, and Lin-Huang Chang. "RSSI-based IPv6 routing metrics for RPL in low-power and lossy networks." In Systems, Man and Cybernetics (SMC), 2014 IEEE International Conference on, pp. 1714-1719. IEEE, 2014.

[7] Alkhatib, Ahmad AA, Gurvinder S. Baicher, and Waleed K. Darwish. "Wireless sensor network-An advanced survey." International Journal of Engineering and Innovative Technology (IJEIT) 2, no. 7 (2013): 355369. 
[8] Xiao, Jiang, Zimu Zhou, Youwen Yi, and Lionel M. Ni. "A survey on wireless indoor localization from the device perspective." ACM Computing Surveys (CSUR) 49, no. 2 (2016): 25.

[9] FIND, available on-line at https://github.com/schollz/find, Visited: April 28th, 2019 at 19:20.

[10] FIND3, available on-line at https://www.internalpositioning.com/doc/, Visited: April, 29th, 2019 at 15:20.

[11] Weng, Chien-Erh, Vishal Sharma, Hsing-Chung Chen, and Chuan-Hsien Mao. "PEER: Proximity-Based Energy-Efficient Routing Algorithm for Wireless Sensor Networks." J. Internet Serv. Inf. Secur. 6, no. 1 (2016): 47-56.

[12] K. Prasanth, P. Sivakumar, "Location Based Routing Protocol-A Survey", 2014 International Conference on Computer Communication and Informatics (ICCCI -2014), Jan. 03 - 05, 2014, Coimbatore, INDIA

[13] Kumar, Arun, Hnin Yu Shwe, Kai Juan Wong, and P. H. Chong. "Location-based routing protocols for wireless sensor networks: A survey." Wireless Sensor Network 9, no. 1 (2017): 25-72.

[14] Camp, Tracy, and Yu Liu. "An adaptive mesh-based protocol for geocast routing." Journal of Parallel and Distributed Computing 63, no. 2 (2003): 196-213.

[15] Li, Wen-Zao, Feng Lin, Ji-Liu Zhou, and Yan Wang. "DTN routing with fixed stations based on the geographic grid approach in an urban environment." Wireless Personal Communications 82, no. 4 (2015): 2033-2049.

[16] Rajaei, Aydin, Dan Chalmers, Ian Wakeman, and George Parisis. "Efficient geocasting in opportunistic networks." Computer Communications 127 (2018): 105-121.

[17] Majumdar, Santanu, P. Rajendra Prasad, S. Santosh Kumar, and KN Sunil Kumar. "An efficient routing algorithm based on ant colony optimisation for VANETs." In 2016 IEEE International Conference on
Recent Trends in Electronics, Information \& Communication Technology (RTEICT), pp. 436-440. IEEE, 2016.

[18] Zahra, Mohamed M. "EFFECT OF ENVIRONMENTS ON GEOCAST ROUTING PROTOCOLS." Journal of Engineering Sciences, Assiut University, Vol. 39, No 6, pp.1417-1429, November 2011.

[19] Boukerche, Azzedine, Begumhan Turgut, Nevin Aydin, Mohammad Z. Ahmad, Ladislau Bölöni, and Damla Turgut. "Routing protocols in ad hoc networks: A survey." Computer networks 55, no. 13 (2011): 30323080.

[20] Yao, Peiling, Ed Krohne, and Tracy Camp. "Performance comparison of geocast routing protocols for a MANET." In Computer Communications and Networks, 2004. ICCCN 2004. Proceedings. 13th International Conference on, pp. 213-220. IEEE, 2004.

[21] Phoummavong, P., Keisuke Utsu, Hiroaki Nishikawa, and Hiroshi Ishii. "Efficient Location-aided Route Discovery Mechanism for Ad-hoc networks." In Proceedings of the International Conference on Parallel and Distributed Processing Techniques and Applications (PDPTA), p. 551. The Steering Committee of the World Congress in Computer Science, Computer Engineering and Applied Computing (WorldComp), 2015.

[22] The VINT Project. The network simulator -ns-2. http://www.isi.edu/nsnam/ns/, Visited: April 28th, 2019 at 19:25.

[23] Pramanik, Aniket, Biplav Choudhury, Tameem S. Choudhury, Wasim Arif, and J. Mehedi. "Simulative study of random waypoint mobility model for mobile ad hoc networks." In 2015 Global Conference on Communication Technologies (GCCT), pp. 112-116. IEEE, 2015.

[24] J. Yoon, M. Liu, and B. Noble. Random waypoint considered harmful. In Proceedings of INFOCOM, pages 1312.1321, 2003.

[25] J. Broch, D. Maltz, D. Johnson, Y. Hu, and J. Jetcheva. Multi-hop wireless ad hoc network routing protocols. In Proceedings of MOBICOM, pages 85.97, 1998. 